\title{
Liver graft from donation after circulatory death donor: Real practice to improve graft viability
}

\author{
Koji Hashimoto \\ Department of General Surgery, Digestive Disease \& Surgery Institute, Cleveland Clinic, Cleveland, OH, USA
}

Donation after circulatory death (DCD) is an increasing source of liver grafts for transplantation, yet outcomes have been inferior compared to donation after brain death liver transplantation. These worse outcomes are mainly due to the severe graft injury resulting from mandatory warm ischemia during DCD organ recovery. New evidence, however, indicates that improved donor selection and surgical techniques can decrease the risk of graft failure and ischemic cholangiopathy (IC). Under current best practices, DCD organs are retrieved with the super-rapid technique, optimizing timing and protecting the liver graft from detrimental warm ischemia. Graft viability is influenced by both the quantity and quality of warm ischemia, which is unique to each donor and causes various degrees of pathophysiologic consequences. Evidence also shows that the choice of preservation solution and premortem heparin administration influences graft viability. Additionally, although the precise mechanism of IC remains unknown, stasis of blood during donor warm ischemia may cause the formation of microthrombi in the peribiliary vascular plexus and ischemia of the bile duct. Importantly, thrombolytic protocols show a possible preventive modality for IC. Finally, while ex vivo machine perfusion technology has gained an interest in DCD liver transplantation, further studies are necessary to evaluate the effectiveness of this evolving field to improve graft quality and transplant outcomes. (Clin Mol Hepatol 2020;26:401-410)

Keywords: Tissue and organ procurement; Liver transplantation; Reperfusion injury; Graft survival; Warm ischemia

\section{INTRODUCTION}

Before the introduction of the brain death law, transplant organs were taken from non-heart beating donors, now commonly referred to as donation after circulatory death (DCD) donors. Due to better outcomes, however, the majority of liver transplantations have been performed using donation after brain death (DBD) donors. With an increasing demand for donor organs, a living donor option was added to mitigate waitlist mortality. To further expand the donor pool, transplant centers were forced to accept donors with increased risks. In the 1990s, DCD donors started regaining attention as a valuable source of liver grafts. Despite growing experiences of DCD in liver transplantation, increased risks of graft failure and ischemic biliary complications remain critical concerns.

The definition of DCD is organ donation from patients who have suffered a catastrophic brain injury but do not meet the criteria for brain death.' In 1995, an international workshop to examine issues surrounding non-heart beating donors was held in Maastricht, Netherlands. The workshop resulted in the classification of DCD donors into four categories: category I (dead on arrival), cat-

\footnotetext{
Abbreviations:

BMI, body mass index; DBD, donation after brain death; DCD, donation after circulatory death; dWIT, donor warm ischemia time; HTK, histidine-tryptophanketoglutarate; IC, ischemic cholangiopathy; IQR, interquartile range; MAP, mean arterial pressure; MELD, Model for End-Stage Liver Disease; OPO, Organ Procurement Organization; $\mathrm{SpO}_{2}$, peripheral oxygen saturation; $\mathrm{PAA}$, tissue plasminogen activator
}

\section{Corresponding author : Koji Hashimoto}

Department of General Surgery, Digestive Disease \& Surgery Institute, Cleveland Clinic, 9500 Euclid Ave, Cleveland, OH 44195, USA

Tel: +1-216-445-0753, Fax: +1-216-444-9357

E-mail: hashimk@ccf.org

https://orcid.org/0000-0002-2327-6777 


\section{CLINCAL and MOLECULAR}

Volume_26 Number_4 October 2020

egory II (unsuccessful resuscitation), category III (awaiting cardiac arrest), and category IV (cardiac arrest in brain dead donor). ${ }^{2}$ Currently, the majority of DCD liver grafts are recovered from category III donors under a controlled environment (controlled DCD). While uncontrolled DCD donors (category II) have been used in some European countries with acceptable outcomes, many transplant centers are reluctant to use them due to unknown warm ischemic time. $^{3}$

Organs recovered from DCD donors inevitably experience warm ischemic injury, which is the primary element distinguishing $D C D$ from DBD (Fig. 1). ${ }^{1}$ A progressive drop in blood pressure and oxygen saturation during an extended withdrawal period is detrimental to graft viability. Since the liver graft is vulnerable to warm ischemic injury, DCD liver transplantation is associated with an increased risk of graft failure and surgical complications. ${ }^{4,5}$ Nonetheless, with the need for liver transplantation is increasing worldwide, DCD donors will continue to be an essential source of lifesaving liver grafts. For the best use of DCD donors, it is crucial to understand the mechanisms of tissue injury of DCD liver grafts and how to maintain graft viability (Table 1). This article reviews the current practice of DCD organ recovery as well as DCD donor selection, which is essential to achieve successful outcomes in
DCD liver transplantation. Further, critical advancement in this field will be discussed regarding preventive modalities for ischemic biliary complications and graft failure.

Table 1. Factors influencing DCD graft viability

\begin{tabular}{lc}
\hline Factor & Reference \\
\hline Donor selection & $6-10$ \\
Donor age & 10 \\
BMl (graft steatosis) & 6,11 \\
Cold ischemia time & \\
Organ recovery & 12 \\
Location of withdrawing life support & 16 \\
Premortem heparin & $17-20$ \\
Preservation solution & \\
Warm ischemia time & $6,22-26$ \\
Extubation to cross clamp & 27,28 \\
Agonal phase & 29 \\
Asystole to cross clamp & $33,39-42$ \\
Thrombolytic agents & $43-49$ \\
\hline Ex vivo machine perfusion
\end{tabular}

$D C D$, donation after circulatory death; BMI, body mass index.

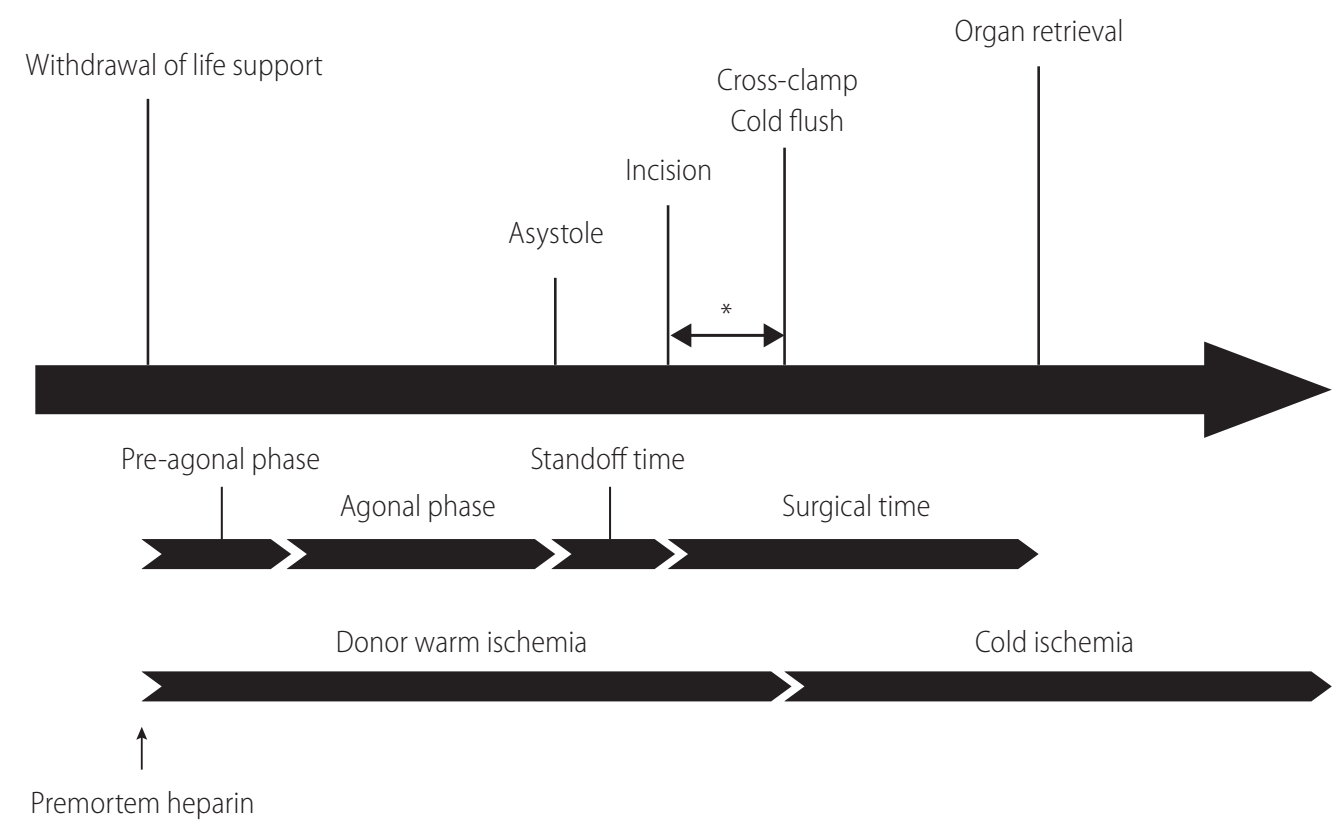

Figure 1. Timeline of DCD organ recovery. The definition of the agonal phase varies based on each program's policy regarding its cutoffs of MAP and $\mathrm{SpO}_{2}$. Generally, the agonal phase begins when blood pressure or $\mathrm{SpO}_{2}$ drops to certain levels $(70-80 \mathrm{mmHg}$ in systolic pressure, $50-60 \mathrm{mmHg}$ in MAP, or $60-80 \%$ in $\mathrm{SpO}_{2}$ ). The standoff (no touch) time lasts at least 2 minutes, but not more than 5 minutes, to confirm the irreversibility of circulatory death. Time from incision to cross-clamp $\left(^{*}\right)$ is the only period that is surgically modifiable to shorten donor warm ischemia. DCD, donation after circulatory death; MAP, mean arterial pressure; $\mathrm{SpO}_{2}$, peripheral oxygen saturation. 


\section{DCD DONOR SELECTION}

Donor age is one of the most potent indicators of graft survival in DCD liver transplantation. According to the Scientific Registry of Transplant Recipients data from September 2001 to April 2009 $(n=1,576)$, donor age of 50-60 years increased the risk of graft failure by $39 \%$ when compared to younger donors. Moreover, the risk jumped to $88 \%$ for donors older than 60 years. ${ }^{6}$ Similarly, based on UK national data on risk factors for graft failure $(n=1,153)$ from January 2000 to December 2015, donor age has been included as an essential element of the DCD donor risk score. $^{7}$

On the other hand, conflicting results have been reported by single-center studies from high volume programs. For instance, a retrospective study by Mayo Clinic showed that recipients who received liver grafts from DCD donors $\geq 50$ years of age achieved comparable graft survival to those who received liver grafts from younger DCD donors. ${ }^{8}$ Our experience at Cleveland Clinic also demonstrated no significant impact of donor age on graft survival with an age cutoff of 45 years. ${ }^{9}$ However, it should be noted that such favorable outcomes could have partly been achieved due to stringent donor and recipient selection criteria. Since the use of DCD donors per se is an independent risk factor, the coexistence of multiple risk factors should be avoided, such as high donor body mass index (BMI), graft steatosis, and high Model for EndStage Liver Disease (MELD) score in recipients. In the Birmingham group's single-center study, an age cutoff of 60 years failed to distinguish recipients with poorer graft and patient survival. ${ }^{10}$ However, when donor age was combined with donor BMI $\geq 25 \mathrm{~kg} / \mathrm{m}^{2}$, graft survival was significantly compromised. ${ }^{10}$ Since high donor BMI is often associated with macrosteatosis in the liver graft, a graft biopsy may be necessary during organ recovery to further evaluate the degree of steatosis. Further, cold ischemia has a significant impact on outcomes in DCD liver transplantation. ${ }^{6,11}$ Indeed, each hour in cold ischemia time increases the risk of graft failure by $6 \% .{ }^{6}$ Accordingly, when accepting aged DCD livers, careful risk assessment is essential to avoid not only donorand recipient-related risks, but also long warm and cold ischemic time. It should also be noted that the use of younger DCD donors does not guarantee a promising outcome, particularly when other unfavorable factors exist. ${ }^{9}$

\section{DCD ORGAN RECOVERY}

\section{End of life care and withdrawal of life support}

After an imminent death is identified, the Organ Procurement Organization (OPO) is notified to initiate an evaluation process to determine whether a patient is suitable to be an organ donor. If the patient does not fulfill the criteria of brain death despite a catastrophic brain injury, a discussion takes place with the family as to whether they wish to proceed with the withdrawal of life support. If the family agrees to proceed, a matched recipient(s) is identified, and an organ recovery team(s) arrives at the donor hospital. Withdrawal of life-sustaining therapy, which includes termination of cardiopulmonary support and all essential medications, often occurs in the operating room or intensive care unit in the presence of the family, depending on the OPO or hospital policy. The family needs to have as much time as needed to say goodbye to their loved one. Generally, the operating room is a preferred location to withdraw life support because the time for transport can be shortened to minimize warm ischemia. Indeed, not only is graft and patient survival better, but the incidence of complications is mitigated when life support is withdrawn in the operating room. ${ }^{12}$ With all comfort care measures in place, the donor is extubated, and life-sustaining treatments are terminated. For the pronouncement of death in DCD organ recovery, the hospital staff must use objective and auditable criteria that are standardized by the law. If the patient does not expire within the time that makes organ donation possible, the process of organ recovery is aborted, and the patient is transferred back to intensive care unit. The donor surgical team needs to wait for at least two minutes, and not more than five minutes, of "standoff time" before starting organ recovery, depending on local policy after donor death is declared (Fig. 1). ${ }^{1}$ This standoff time is vital to confirm the irreversibility of circulatory death. It should be noted that operating room personnel who will be involved in organ recovery or transplantation must not be present when withdrawal of life support takes place.

\section{Organ recovery by super-rapid technique}

After the required standoff time, the surgical team begins organ recovery with a midline incision from the suprasternal notch to the symphysis pubis. ${ }^{13}$ After placing a Balfour retractor, an assistant holds the small intestine upward to expose the sacral promontory. At approximately $5 \mathrm{~cm}$ superior to the sacral promontory, which 
may vary based on age, gender, and race, the bifurcation of the aorta can be identified in the retroperitoneum. ${ }^{14}$ It is crucial to stay at the midline to avoid inadvertent injury of the ureters. The distal aorta is incised to insert a cannula to commence cold perfusion (Fig. 2). Then, the inferior vena cava next to the aorta is incised to vent blood. A pool suction tip is inserted into the vena cava to assist venting. Next, the sternum is split, and the thoracic aorta is cross-clamped. Finally, the pericardium is opened, and the inferior vena cava is incised below the right atrium to decompress the transplant organs further. The abdominal cavity is then filled with ice slush. If a thoracic team is present, the supraceliac aorta, instead of the thoracic aorta, is cross-clamped between the esophagus and the caudate lobe of the liver. When the lesser sac is opened to gain access to the aorta, special attention should be paid to avoid inadvertent injury to the left accessory hepatic artery. While the inferior mesenteric vein can be cannulated to perfuse the portal system, there is no evidence that this improves graft quality in DCD. Once core cooling is achieved, the liver is recovered using the same technique for DBD organ recovery. With an experienced surgical team, the process from skin incision to cross-clamp can be done within a few minutes.

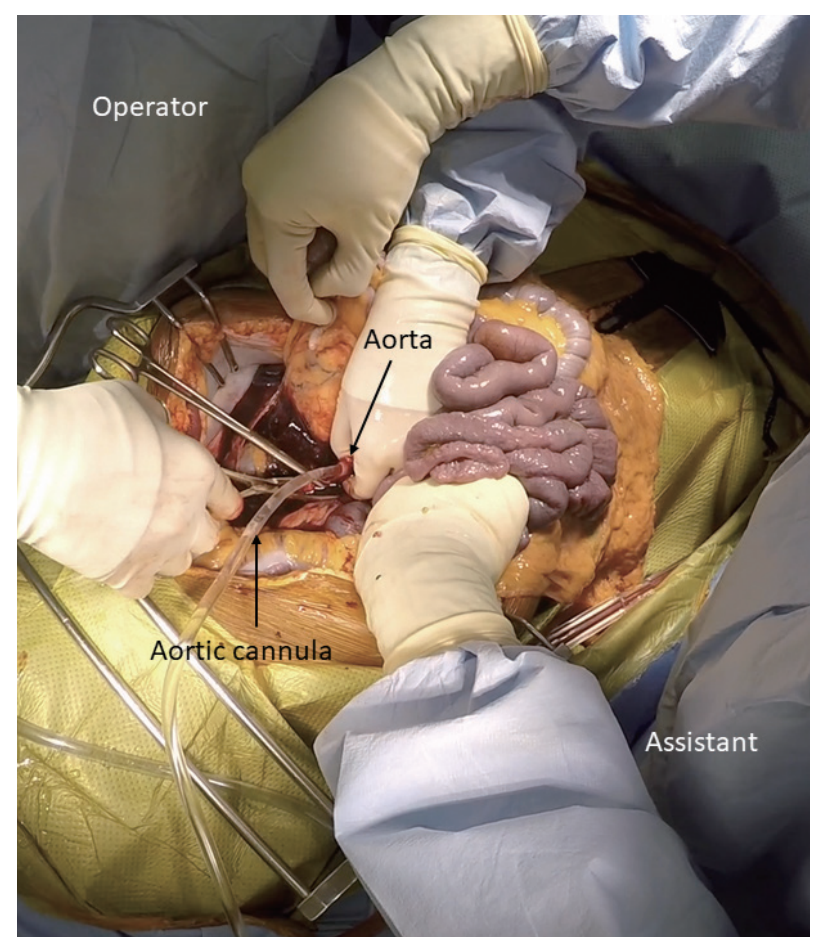

Figure 2. DCD organ recovery with the super-rapid technique. Aortic cannulation is done to commence cold perfusion while the assistant holds the small intestine upward to expose the retroperitoneum. DCD, donation after circulatory death.

\section{Premortem heparin administration}

Systemic administration of heparin is universally adopted in DBD organ recovery to prevent thrombus formation in transplanted organs. In DCD donors, however, premortem heparin administration $(30,000$ units) is ethically controversial because systemic heparinization may hasten the death of donors due to theoretical concerns of intracranial hemorrhage or worsening of bleeding. $.^{15} \mathrm{Ac}-$ cording to the Scientific Registry of Transplant Recipients data, premortem heparin is currently used in $>90 \%$ of DCD donors as a critical component of the DCD protocol. ${ }^{16}$ When premortem heparin is prohibited due to a local policy, heparin can be mixed in an initial bag of the cold preservation solution. Without premortem heparin, the risks of graft failure and primary non-function increase by $18 \%$ and $81 \%$, respectively. ${ }^{16}$ Premortem heparin does not improve the discard rate of DCD livers. $^{16}$

\section{Preservation solution}

The selection of preservation solutions may be critical to maintaining the viability of DCD livers. Conflicting data exists regarding the comparison between University of Wisconsin solution versus histidine-tryptophan-ketoglutarate (HTK) solution in DCD liver transplantation. ${ }^{17-19}$ Early studies failed to demonstrate significant differences in graft survival when comparing these solutions, possibly due to the small sample size. According to data from the United Network for Organ Sharing database from July 2004 to February 2008, however, the use of HTK solution was associated with an increased risk of graft failure by $44 \%$ in DCD grafts compared to University of Wisconsin solution $(P=0.025)$, especially when cold ischemia time was $>8$ hours. ${ }^{20}$ Despite the data, some transplant centers prefer HTK solution due to lower viscosity, which might be beneficial to prevent biliary complications. ${ }^{17}$

\section{QUANTITY AND QUALITY OF DONOR WARM ISCHEMIA TIME}

In early experience, Feng et al. ${ }^{21}$ reported that the use of DCD donors was one of the most substantial risk factors for liver graft failure. Compared to DBD, the inferiority of DCD mainly stems from a poorer quality of liver grafts. Donor warm ischemia time (dWIT) is generally defined as the time from extubation to crossclamp (Fig. 1), the length of which has been considered a potent indicator of poor outcomes. ${ }^{22-24}$ Since early experience has dem- 
onstrated that prolonged dWIT is associated with a lower graft survival and an increased risk of biliary complications, many transplant centers have adopted 30 minutes of dWIT as a cutoff to accept DCD livers. Indeed, the Scientific Registry of Transplant Recipients data demonstrated that the risk of graft loss increased by $84 \%$ when dWIT was $\geq 35$ minutes. ${ }^{6}$ However, many studies have shown an equivocal association between dWIT and transplant outcomes. ${ }^{25-28}$ Such conflicting findings are mainly attributable to the heterogeneity of dWIT. Namely, from the withdrawal of life support to circulatory death, a wide range of hemodynamic perturbations is seen among donors. Changes in tissue perfusion and oxygenation during the agonal phase are not equal in each donor. Therefore, the trajectory of donor hemodynamics should be considered as an essential parameter to assess DCD liver graft quality.

During DCD organ recovery, the donor surgical team receives a minute-by-minute update of donor hemodynamics, including blood pressure, oxygen saturation, and heart rate. Peripheral arterial pressure and peripheral oxygen saturation $\left(\mathrm{SpO}_{2}\right)$ are closely related to graft quality, and are particularly important. It is essential to understand that the time from withdrawal of life support to asystole consists of the pre-agonal and agonal phases (Fig. 1). Generally, the agonal phase begins when blood pressure or $\mathrm{SpO}_{2}$ drops to certain levels $(70-80 \mathrm{mmHg}$ in systolic pressure, $50-60$ $\mathrm{mmHg}$ in mean arterial pressure [MAP], or $60-80 \%$ in $\mathrm{SpO}_{2}$ ) in DCD organ recovery. During the agonal phase, donors become progressively hypotensive and hypoxic. However, no donor has an identical change of these hemodynamic parameters to others, and the exact timing of the onset of liver graft injury is unknown (Fig. 3).

Based on the clinical data of 87 DCD donors at Cleveland Clinic, trajectories of MAP were categorized into three clinically meaningful phenotypes (Fig. 4). ${ }^{28}$ The patient group who had the worst graft survival (1-year graft survival, 68.9\%) was the group of patients who received liver grafts from Cluster 1 donors. With minimal or short pre-agonal phase, MAP declined gradually to asystole with a prolonged agonal phase ( $\geq 15$ minutes, slow decliner) (Figs. 4, 5). The median dWIT of Cluster 1 was 27 minutes (interquartile range [IQR], 24-30 minutes). While dWIT was comparable (median, 29 minutes; IQR, 25-32 minutes), Cluster 2 donors maintained stable MAP during the pre-agonal phase over $10 \mathrm{~min}$ utes, which was followed by a rapid decline with a short agonal phase ( $<15$ minutes) (Fig. 4). Finally, Cluster 3 donors had the shortest dWIT (median, 19 minutes; IQR, 16-22 minutes; $P<0.001$ vs. Cluster 1 and 2) with a rapid decline of MAP from withdrawal to asystole (agonal phase $<15$ minutes). Interestingly and despite the longer dWIT, recipients who received Cluster 2 liver grafts had comparable graft survival to recipients with Cluster 3 donors (1year graft survival, $90.9 \%$ in Cluster 2 and $91.3 \%$ in Cluster 3). This favorable outcome in Cluster 2 is probably due to the pre-agonal phase of dWIT, which appears to help maintain adequate perfusion and oxygenation of the liver graft. With a similar phenotype in the agonal phase, Cluster 2 and 3 donors can be categorized as the rapid decliner, who has better graft survival than the slow decliner (Figs. 4, 5). The difference between these phenotypes indicates that both the quantity (length) and quality of dWIT play a crucial role in determining graft survival. Similar findings were seen with $\mathrm{SpO}_{2}$ trajectories. ${ }^{28}$

Taner et al. ${ }^{29}$ reported that time from asystole to cross-clamp was important to determine the viability of DCD liver grafts. This
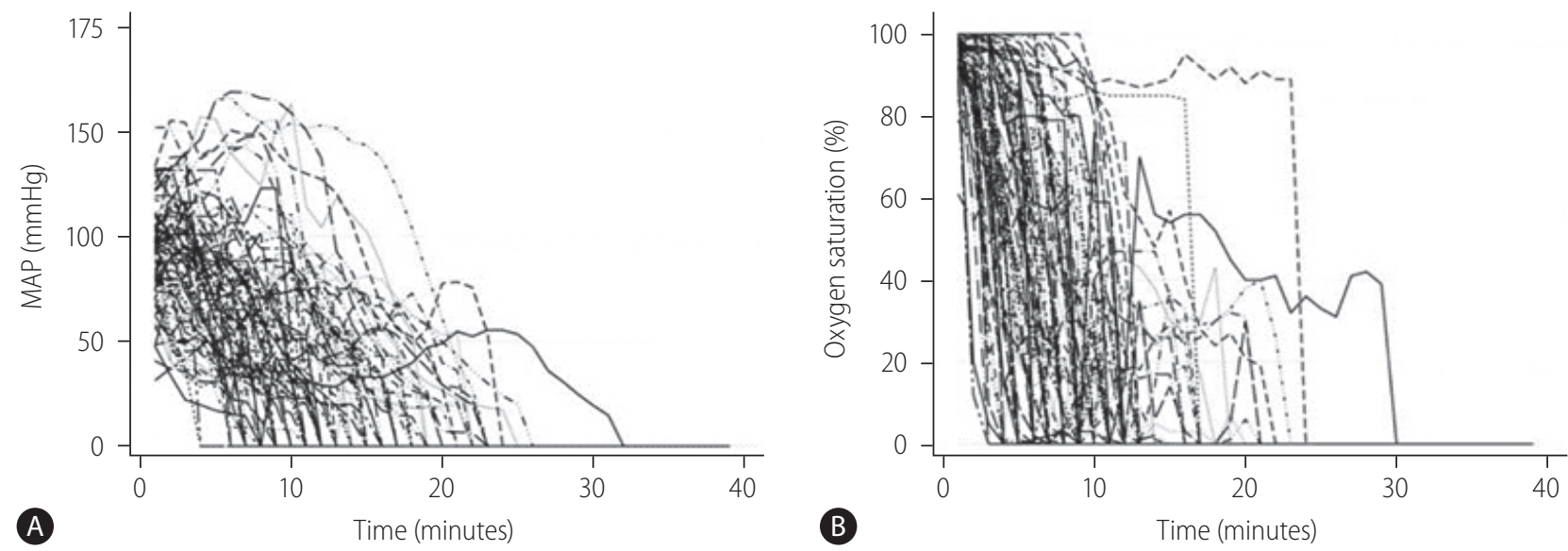

Figure 3. Hemodynamic trajectories in DCD donors $(n=87)$ from the time of withdrawal of life support to asystole by $(A) M_{A P}$ and $(B) S p O_{2}$. Adapted from Firl et al. ${ }^{28}$ with permission. DCD, donation after circulatory death; $\mathrm{MAP}$, mean arterial pressure; $\mathrm{SpO}_{2}$, peripheral oxygen saturation. 


\section{CLINICAL and MOLECULAR}

\section{HEPATOLOGY}

asystole-cross clamp time consists of two critical periods, including the first portion as standoff time ( $2-5$ minutes) to confirm the irreversibility of circulatory death, and the second portion which is the surgical time from incision to cross-clamp (Fig. 1). Both periods are critical because the liver graft receives no perfusion or oxygenation in the donor body due to stasis of blood. To shorten this asystole-cross clamp period, mandatory standoff time can be minimized to 2 minutes as recommended by the American Society of Transplant Surgeons. 'However, it is also imperative to maintain public confidence in the irreversibility of death determination. Since the second portion is the only time that is surgically modifiable, it is essential for the surgical team to commence cold perfusion and cross-clamp as quickly as possible (Fig. 1).

\section{ISCHEMIC CHOLANGIOPATHY (IC)}

Increased risk of biliary complications is the Achilles' heel of DCD liver transplantation. IC causes diffuse or focal intrahepatic biliary stricture, liver abscess, and necrosis, resulting in graft failure and retransplantation. The incidence of IC in DCD liver transplantation has been reported to be as high as $30-50 \%$, and is one of the reasons transplant centers hesitate to accept DCD donors. ${ }^{17,22,25,30,31}$ Patients who develop IC have significantly inferior graft survival compared to those who do not, because there is no effective therapeutic modality to salvage liver grafts from IC. Therefore, it is vital to prevent the development of IC.

The precise mechanism for the development of IC remains un-
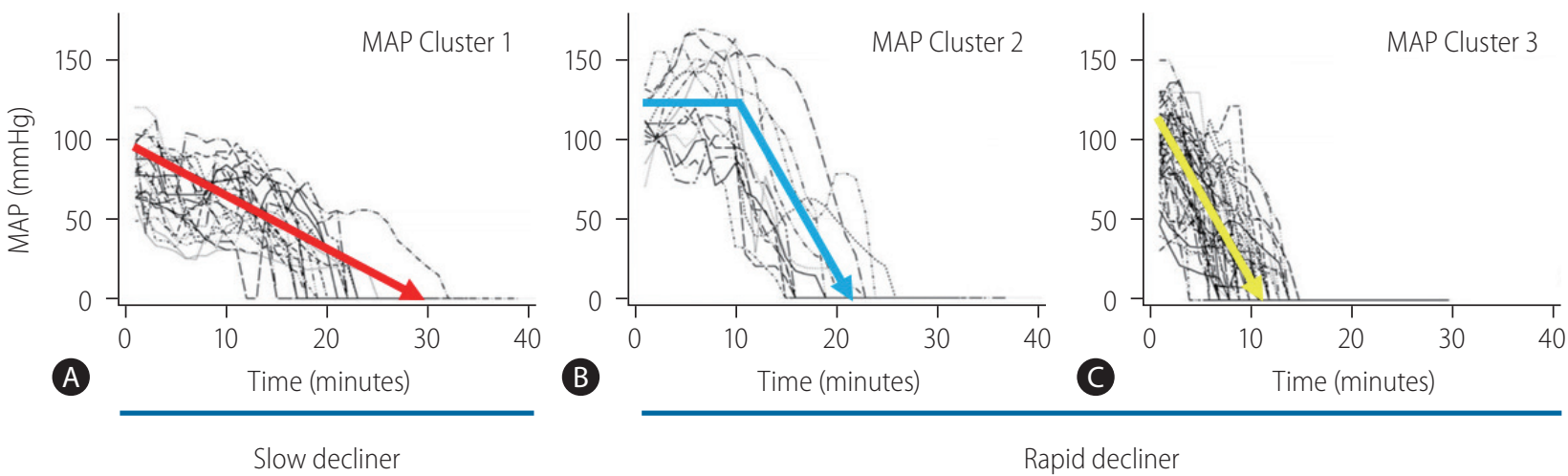

Rapid decliner

Figure 4. Individual donor MAP trajectories shown by (A) Cluster 1 ( $n=24)$, (B) Cluster 2 ( $n=14$ ), and (C) Cluster 3 ( $n=49)$. Cluster 1 donors are defined as the slow decliner due to a prolonged agonal phase ( $\geq 15$ minutes) with a gradual MAP decline. On the other hand, Cluster 2 and Cluster 3 are defined as the rapid decliner because their agonal phases last less than 15 minutes. Cluster 2 donors maintain stable MAP over 10 minutes during the pre-agonal phase after withdrawal of life support. Adapted from Firl et al. ${ }^{28}$ with permission. MAP, mean arterial pressure.

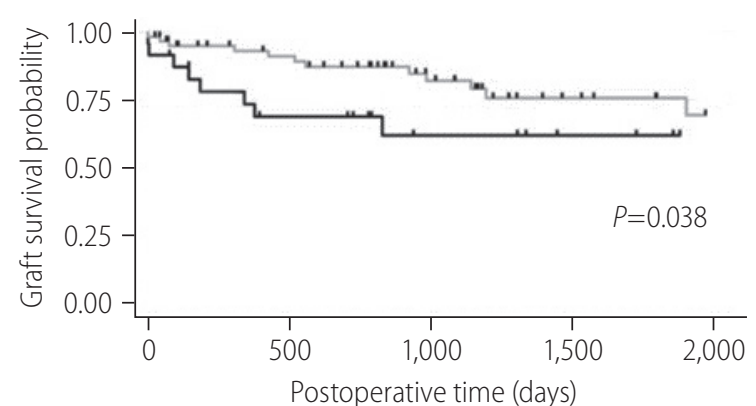

Number at risk Slow decliner 24 Rapid decliner 63

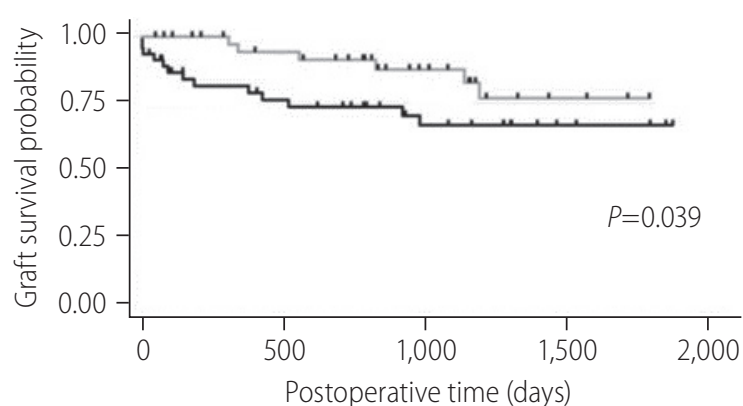

Number at risk

Slow decliner 46

Rapid decliner 41

$\begin{array}{lllll}46 & 29 & 19 & 11 & 7 \\ 41 & 32 & 20 & 10 & 5\end{array}$

$\mathrm{SpO}_{2}$ slow decliner $-\mathrm{SpO}_{2}$ rapid decliner

Figure 5. Graft survival of the slow decliner (Cluster 1 ) and rapid decliner (Cluster 2 and 3) with respect to (A) MAP $(P=0.038)$ and (B) SpO $(P=0.039)$. The rapid decliner had significantly better graft survival than the slow decliner. Adapted from Firl et al. ${ }^{28}$ with permission. MAP, mean arterial pressure; $\mathrm{SpO}_{2}$, peripheral oxygen saturation. 
known. Interestingly, clinical and radiological presentations of IC are similar to those of hepatic artery thrombosis. The peribiliary vascular plexus of the human liver receives blood supply only via the hepatic artery. ${ }^{32,33}$ Therefore, a similar ischemic etiology may play a role in the development of IC. If microthrombi are formed in the peribiliary vascular plexus due to stasis of blood during warm ischemia, microcirculation of the intrahepatic bile duct can be disturbed. If this is the case, the formation of microthrombi causes ischemic biliary necrosis (Fig. 6). ${ }^{33}$

Study results offer conflicting evidence regarding microthrombi formation in DCD livers. An animal model demonstrated an experimental evidence of microthrombi formation in the peribiliary vascular plexus. ${ }^{34} \mathrm{~A}$ human study also demonstrated that peribiliary vascular injury with microthrombi is more prominent in $D C D$ compared with DBD liver grafts, which correlates with the incidence of IC. ${ }^{35}$ On the other hand, Verhoeven et al. ${ }^{36}$ reported no evidence of increased microthrombi formation in human DCD liver grafts.

An early study from the Pittsburgh group demonstrated the usefulness of thrombolytic therapy with surgical revascularization to rescue liver grafts from hepatic artery thrombosis. ${ }^{37}$ When such urgent intervention is conducted before irreversible tissue damage occurs, thrombolytic therapy can help prevent biliary necrosis. ${ }^{38}$ According to these clinical findings, the use of thrombolytic agents may be of benefit to prevent the development of IC.

In August 2005, Cleveland Clinic implemented a new DCD protocol to use tissue plasminogen activator (tPA) to prevent IC. The protocol was used in 68 patients until December 2013; 22 patients in an initial pilot study ${ }^{33}$ and 46 patients in a following pro- spective study. ${ }^{39}$ Assuming the formation of microthrombi is the mechanism of IC, tPA (Activase ${ }^{\circledR} 1 \mathrm{mg} / \mathrm{mL}, 0.5 \mathrm{mg}$ per $100 \mathrm{~g}$ liver weight; Genentech Inc., South San Francisco, CA, USA) was injected into the donor hepatic artery on the back table. After portal reperfusion, the hepatic artery was kept clamped for 10-15 minutes. To minimize the systemic introduction of tPA, the hepatic artery was unclamped before arterial anastomosis to allow excess tPA to back-bleed and discard the effluent. In the initial pilot study, the protocol dose of tPA was used in 12 grafts. Reduced doses (0.2-0.4 mg per $100 \mathrm{~g}$ liver weight) were used in 10 grafts based on the presence of various risk factors for bleeding such as donor age, dWIT, cold ischemia time, MELD score, and previous history of major laparotomy in recipients. Among the 22 patients in the pilot study, only two recipients (9\%) developed IC, including one with diffuse necrosis requiring retransplantation and another with focal stricture of the intrahepatic bile duct with a functioning graft. ${ }^{33}$ However, the randomized prospective study failed to demonstrate a preventive effect of tPA for IC (7\% [3/46] with tPA vs. 3\% [1/33] without tPA, $P=0.6)$. Although there was a trend toward lower incidence of early allograft dysfunction with tPA (17\% vs. 34\%, $P=0.07)$, there was no difference in the incidence of primary non-function, hepatic artery thrombosis, and retransplantation. ${ }^{39}$ Since 2014, Cleveland Clinic abandoned the thrombolytic protocol based on the results of the prospective study, as well as the low incidence of IC even without the tPA treatment.

University of Toronto and Ochsner Clinic also adopted a new thrombolytic protocol. ${ }^{40,41}$ With the Ochsner Clinic protocol, $2 \mathrm{mg}$ of tPA was injected after $5 \mathrm{mg}$ of verapamil into the donor hepatic
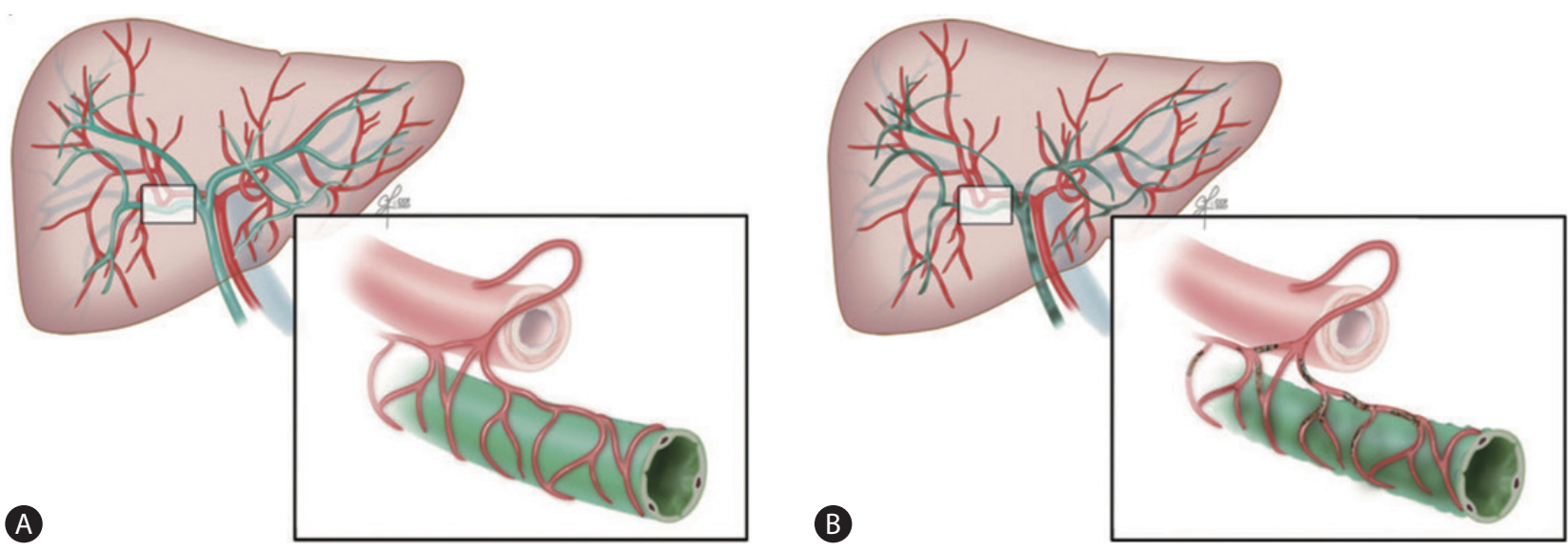

Figure 6. The postulated mechanism of the development of IC in DCD liver transplantation. (A) The blood supply to the human biliary system depends solely on the hepatic artery via the peribiliary vascular plexus. (B) Formation of microthrombi in the peribiliary vascular plexus during donor warm ischemia may contribute to biliary ischemia. Adapted from Hashimoto et al. ${ }^{33}$ with permission. DCD, donation after circulatory death. 
artery after portal vein anastomosis. The University of Toronto protocol injected tPA (100 $\mu \mathrm{g} / \mathrm{kg}$ donor body weight) into the donor hepatic artery before portal vein anastomosis. While $21.2 \%$ of patients at Ochsner Clinic and the University of Toronto experienced IC without tPA ( $n=33$ ), IC occurred in less than $3.5 \%$ of patients with the tPA protocols ( $n=56$ at Ochsner Clinic, $n=29$ at University of Toronto; $P<0.005) .{ }^{40} \mathrm{~A}$ subsequent study by Ochsner Clinic with 100 DCD liver transplantations demonstrated that the tPA protocol achieved comparable graft and patient survival compared with DBD liver transplantation, with an IC incidence of only $3 \% .{ }^{41}$ Indiana University developed a different thrombolytic protocol along with a systematic approach, including shorter donor and recipient ischemia time, as well as shorter operation time. ${ }^{42}$ In their protocol, $100 \mathrm{mg}$ of tPA was mixed with $1 \mathrm{~L}$ of room temperature normal saline, which was administered via the donor aorta as an initial flush solution in organ recovery. This protocol achieved a zero incidence of IC in 30 patients who received DCD liver grafts.

Due to the risk of excessive bleeding, transplant centers are generally reluctant to adopt the use of thrombolytic agents. Yet results from the Centers that used these existing thrombolytic protocols do not support this concern. ${ }^{33,40-42}$ Still, since coagulopathy, fibrinolysis, and thrombocytopenia are not uncommon in DCD liver transplantation, the risk of bleeding should not be underestimated even with the small dose of thrombolytic agents. ${ }^{33}$

\section{EX VIVO MACHINE PERFUSION}

Ex vivo machine perfusion technology has recently gained the increased interest in DCD liver transplantation. Several single-center studies in the hypothermic setting have demonstrated less graft tissue damage, better graft survival, and lower incidence of IC, compared with static cold preservation. ${ }^{43-45}$ On the other hand, normothermic machine perfusion, which creates more physiologic conditions to preserve, assess, and potentially repair liver grafts, is expected to improve outcomes for DCD liver transplantation even further. To date, however, clinical data are not available to support normothermic machine perfusion as a preventive and therapeutic modality. ${ }^{46-48}$ While a randomized clinical trial of normothermic machine perfusion achieved a $50 \%$ reduction of graft injury, the study demonstrated no significant difference in survival or IC rates compared to static cold preservation. ${ }^{49}$ It should be noted, however, that normothermic machine perfusion helped decrease the organ discard rate, providing an opportunity to in- crease the donor pool. ${ }^{49}$ Future studies are necessary to further explore the potential of this evolving technology in terms of graft viability and ex vivo organ repair.

\section{CONCLUSION}

In real practice, the DCD liver graft increases access to lifesaving organs for patients awaiting liver transplantation. While the increased risks of graft failure and IC are of concern, outcomes after DCD liver transplantation have improved over the last two decades with a better understanding of donor selection and organ recovery techniques. It is vital to understand the importance of not only the quantity but also the quality of donor warm ischemia to determine DCD graft viability because each donor has different nature of warm ischemia, which induces various degrees of pathophysiologic consequences. Potential interventions, using thrombolytic agents and ex vivo machine perfusion technology, are evolving with encouraging reports from major transplant centers. Continued research is necessary to optimize existing protocols as well as develop new therapeutic approaches.

\section{Acknowledgements}

The author would like to acknowledge Ms. Sally Garrett Karyo for her editorial assistance.

\section{Conflicts of Interest}

The author has no conflicts to disclose.

\section{REFERENCES}

1. Reich DJ, Mulligan DC, Abt PL, Pruett TL, Abecassis MM, D'Alessandro $A$, et al. ASTS recommended practice guidelines for controlled donation after cardiac death organ procurement and transplantation. Am J Transplant 2009;9:2004-2011.

2. Kootstra G, Daemen JH, Oomen AP. Categories of non-heart-beating donors. Transplant Proc 1995;27:2893-2894.

3. Fondevila C, Hessheimer AJ, Flores E, Ruiz A, Mestres N, Calatayud $D$, et al. Applicability and results of Maastricht type 2 donation after cardiac death liver transplantation. Am J Transplant 2012;12:162170.

4. Laing RW, Scalera I, Isaac J, Mergental H, Mirza DF, Hodson J, et al. Liver transplantation using grafts from donors after circulatory death: a propensity score-matched study from a single center. Am J Transplant 2016;16:1795-1804. 
5. Croome KP, Lee DD, Perry DK, Burns JM, Nguyen JH, Keaveny AP, et al. Comparison of longterm outcomes and quality of life in recipients of donation after cardiac death liver grafts with a propensitymatched cohort. Liver Transpl 2017;23:342-351.

6. Mathur AK, Heimbach J, Steffick DE, Sonnenday CJ, Goodrich NP, Merion RM. Donation after cardiac death liver transplantation: predictors of outcome. Am J Transplant 2010;10:2512-2519.

7. Schlegel A, Kalisvaart M, Scalera I, Laing RW, Mergental H, Mirza $D F$, et al. The UK DCD Risk Score: a new proposal to define futility in donation-after-circulatory-death liver transplantation. J Hepatol 2018;68:456-464

8. Croome KP, Mathur AK, Lee DD, Moss AA, Rosen CB, Heimbach JK, et al. Outcomes of donation after circulatory death liver grafts from donors 50 years or older: a multicenter analysis. Transplantation 2018;102:1108-1114.

9. Firl DJ, Hashimoto K, O'Rourke C, Diago-Uso T, Fujiki M, Aucejo FN, et al. Impact of donor age in liver transplantation from donation after circulatory death donors: a decade of experience at Cleveland Clinic. Liver Transpl 2015;21:1494-1503.

10. Schlegel A, Scalera I, Perera MTPR, Kalisvaart M, Mergental H, Mirza DF, et al. Impact of donor age in donation after circulatory death liver transplantation: is the cutoff " 60 " still of relevance? Liver Transpl 2018;24:352-362.

11. Lee KW, Simpkins CE, Montgomery RA, Locke JE, Segev DL, Maley WR. Factors affecting graft survival after liver transplantation from donation after cardiac death donors. Transplantation 2006;82:16831688.

12. Cao Y, Shahrestani S, Chew HC, Crawford M, Macdonald PS, Laurence J, et al. Donation after circulatory death for liver transplantation: a meta-analysis on the location of life support withdrawal affecting outcomes. Transplantation 2016;100:1513-1524.

13. Casavilla A, Ramirez C, Shapiro R, Nghiem D, Miracle K, Bronsther 0 , et al. Experience with liver and kidney allografts from non-heartbeating donors. Transplantation 1995;59:197-203.

14. Agrawal A, Abayazeed A, Francis SL, Tolentino J, Ostergard DR, Seow $A$, et al. Correlation of patient age with CT-measured aortasacral promontory distance. Int Urogynecol J 2015;26:887-891.

15. DuBois JM, Delmonico FL, D'Alessandro AM. When organ donors are still patients: is premortem use of heparin ethically acceptable? Am J Crit Care 2007;16:396-400.

16. Narvaez JRF, Nie J, Noyes K, Kayler LK. Transplant outcomes of donation after circulatory death livers recovered with versus without premortem heparin administration. Liver Transpl 2020;26:247-255.

17. Fung JJ, Eghtesad B, Patel-Tom K. Using livers from donation after cardiac death donors--a proposal to protect the true Achilles heel. Liver Transpl 2007;13:1633-1636.

18. Mangus RS, Fridell JA, Vianna RM, Milgrom MA, Chestovich P, Chihara RK, et al. Comparison of histidine-tryptophan-ketoglutarate solution and University of Wisconsin solution in extended criteria liver donors. Liver Transpl 2008;14:365-373.

19. Gulsen MT, Girotra M, Cengiz-Seval G, Price J, Singh VK, Segev DL, et al. HTK preservative solution is associated with increased biliary complications among patients receiving DCD liver transplants: a single center experience. Ann Transplant 2013;18:69-75.

20. Stewart ZA, Cameron AM, Singer AL, Montgomery RA, Segev DL. Histidine-Tryptophan-Ketoglutarate (HTK) is associated with reduced graft survival in deceased donor livers, especially those donated after cardiac death. Am J Transplant 2009;9:286-293.

21. Feng S, Goodrich NP, Bragg-Gresham JL, Dykstra DM, Punch JD, DebRoy MA, et al. Characteristics associated with liver graft failure: the concept of a donor risk index. Am J Transplant 2006;6:783-790.

22. Abt P, Crawford M, Desai N, Markmann J, Olthoff K, Shaked A. Liver transplantation from controlled non-heart-beating donors: an increased incidence of biliary complications. Transplantation 2003;75:1659-1663.

23. Mateo R, Cho Y, Singh G, Stapfer M, Donovan J, Kahn J, et al. Risk factors for graft survival after liver transplantation from donation after cardiac death donors: an analysis of OPTN/UNOS data. Am J Transplant 2006;6:791-796.

24. de Vera ME, Lopez-Solis R, Dvorchik I, Campos S, Morris W, Demetris AJ, et al. Liver transplantation using donation after cardiac death donors: long-term follow-up from a single center. Am J Transplant 2009;9:773-781.

25. Chan EY, Olson LC, Kisthard JA, Perkins JD, Bakthavatsalam R, Halldorson JB, et al. Ischemic cholangiopathy following liver transplantation from donation after cardiac death donors. Liver Transpl 2008;14:604-610.

26. Foley DP, Fernandez LA, Leverson G, Anderson M, Mezrich J, Sollinger HW, et al. Biliary complications after liver transplantation from donation after cardiac death donors: an analysis of risk factors and long-term outcomes from a single center. Ann Surg 2011;253:817-825.

27. Abt PL, Praestgaard J, West S, Hasz R. Donor hemodynamic profile presages graft survival in donation after cardiac death liver transplantation. Liver Transpl 2014;20:165-172.

28. Firl DJ, Hashimoto K, O'Rourke C, Diago-Uso T, Fujiki M, Aucejo FN, et al. Role of donor hemodynamic trajectory in determining graft survival in liver transplantation from donation after circulatory death donors. Liver Transpl 2016;22:1469-1481.

29. Taner CB, Bulatao IG, Perry DK, Sibulesky L, Willingham DL, Kramer $D J$, et al. Asystole to cross-clamp period predicts development of biliary complications in liver transplantation using donation after cardiac death donors. Transpl Int 2012;25:838-846.

30. Maheshwari A, Maley W, Li Z, Thuluvath PJ. Biliary complications and outcomes of liver transplantation from donors after cardiac death. Liver Transpl 2007;13:1645-1653. 
31. Skaro Al, Jay CL, Baker TB, Wang E, Pasricha S, Lyuksemburg V, et al. The impact of ischemic cholangiopathy in liver transplantation using donors after cardiac death: the untold story. Surgery 2009;146:543-552; discussion 552-553.

32. Yamamoto K, Sherman I, Phillips MJ, Fisher MM. Three-dimensional observations of the hepatic arterial terminations in rat, hamster and human liver by scanning electron microscopy of microvascular casts. Hepatology 1985;5:452-456.

33. Hashimoto K, Eghtesad B, Gunasekaran G, Fujiki M, Uso TD, Quintini $C$, et al. Use of tissue plasminogen activator in liver transplantation from donation after cardiac death donors. Am J Transplant 2010;10:2665-2672.

34. Du Z, Dong $S$, Lin $P$, Chen $S$, Wu S, Zhang $S$, et al. Warm ischemia may damage peribiliary vascular plexus during DCD liver transplantation. Int J Clin Exp Med 2015;8:758-763.

35. op den Dries $S$, Westerkamp AC, Karimian N, Gouw AS, Bruinsma $B G$, Markmann JF, et al. Injury to peribiliary glands and vascular plexus before liver transplantation predicts formation of non-anastomotic biliary strictures. J Hepatol 2014;60:1172-1179.

36. Verhoeven CJ, Simon TC, de Jonge J, Doukas M, Biermann K, Metselaar $\mathrm{HJ}$, et al. Liver grafts procured from donors after circulatory death have no increased risk of microthrombi formation. Liver Transpl 2016;22:1676-1687.

37. Pinna AD, Smith CV, Furukawa $H$, Starzl TE, Fung JJ. Urgent revascularization of liver allografts after early hepatic artery thrombosis. Transplantation 1996;62:1584-1587.

38. Fujiki M, Hashimoto K, Palaios E, Quintini C, Aucejo FN, Uso TD, et al. Probability, management, and long-term outcomes of biliary complications after hepatic artery thrombosis in liver transplant recipients. Surgery 2017;162:1101-1111.

39. Eghtesad B, Hashimoto K, Watson M, Nazal M, Quintini C, Kelly D, et al. Use of tissue plasminogen activator (TPA) in liver transplantation from donation after cardiac death (DCD) donors: a controlled randomized trial. Am J Transplant 2015;15:275.

40. Seal JB, Bohorquez H, Reichman T, Kressel A, Ghanekar A, Cohen A, et al. Thrombolytic protocol minimizes ischemic-type biliary complications in liver transplantation from donation after circulatory death donors. Liver Transpl 2015;21:321-328.

41. Bohorquez H, Seal JB, Cohen AJ, Kressel A, Bugeaud E, Bruce DS, et al. Safety and outcomes in 100 consecutive donation after circulatory death liver transplants using a protocol that includes thrombolytic therapy. Am J Transplant 2017;17:2155-2164.

42. Kubal C, Mangus R, Fridell J, Saxena R, Rush N, Wingler M, et al. Optimization of perioperative conditions to prevent ischemic cholangiopathy in donation after circulatory death donor liver transplantation. Transplantation 2016;100:1699-1704.

43. Dutkowski P, Polak WG, Muiesan P, Schlegel A, Verhoeven CJ, Scalera I, et al. First comparison of hypothermic oxygenated perfusion versus static cold storage of human donation after cardiac death liver transplants: an international-matched case analysis. Ann Surg 2015;262:764-770; discussion 770-771.

44. van Rijn R, Karimian N, Matton APM, Burlage LC, Westerkamp AC, van den Berg AP, et al. Dual hypothermic oxygenated machine perfusion in liver transplants donated after circulatory death. Br J Surg 2017;104:907-917.

45. Schlegel A, Muller X, Kalisvaart M, Muellhaupt B, Perera MTPR, Isaac JR, et al. Outcomes of DCD liver transplantation using organs treated by hypothermic oxygenated perfusion before implantation. J Hepatol 2019;70:50-57.

46. Ravikumar R, Jassem W, Mergental H, Heaton N, Mirza D, Perera $M T$, et al. Liver transplantation after ex vivo normothermic machine preservation: a phase 1 (first-in-man) clinical trial. Am J Transplant 2016;16:1779-1787.

47. Selzner M, Goldaracena N, Echeverri J, Kaths JM, Linares I, Selzner $N$, et al. Normothermic ex vivo liver perfusion using steen solution as perfusate for human liver transplantation: First North American results. Liver Transpl 2016;22:1501-1508.

48. Bral M, Gala-Lopez B, Bigam D, Kneteman N, Malcolm A, Livingstone $\mathrm{S}$, et al. Preliminary single-center Canadian experience of human normothermic ex vivo liver perfusion: results of a clinical trial. Am J Transplant 2017:17:1071-1080.

49. Nasralla D, Coussios CC, Mergental H, Akhtar MZ, Butler AJ, Ceresa $C D L$, et al. A randomized trial of normothermic preservation in liver transplantation. Nature 2018;557:50-56. 